\title{
Convergence rate of maxima of bivariate Gaussian arrays to the Hüsler-Reiss distribution
}

\author{
Xin Liao And Zuoxiang PenG*
}

The limit distribution of maxima formed by a triangular array of independent and identically distributed bivariate Gaussian random vectors is the Hüsler-Reiss max-stable distribution if and only if the correlation of each vector approaches one with a certain rate. In this paper, we introduce a second-order condition on the convergence rate of this correlation. Under this condition we derive the uniform convergence rate of the distribution of normalized bivariate maxima to its ultimate limit distribution.

AMS 2000 SubJeCt Classifications: Primary 62E20, 60G70; secondary 60F15, 60F05.

KEYWORDS AND PHRASES: Bivariate Gaussian random vector, Hüsler-Reiss max-stable distribution, Maximum, Uniform convergence rate.

\section{INTRODUCTION}

Let $\left\{\left(\xi_{n i}, \eta_{n i}\right), 1 \leq i \leq n, n \geq 1\right\}$ be a triangular array of bivariate Gaussian random vectors, which are independent for each fixed $n$. For a given $n \geq 1$, let $F(x, y)$ denote the bivariate Gaussian distribution function of $\left(\xi_{n i}, \eta_{n i}\right)$, and the correlation coefficient of unit Gaussian distributed $\xi_{n i}$ and $\eta_{n i}$ is represented by $\rho_{n}, 1 \leq i \leq n$. The bivariate maxima $\mathbf{M}_{n}$ is defined componentwise by

$$
\mathbf{M}_{n}=\left(M_{n 1}, M_{n 2}\right)=\left(\max _{1 \leq i \leq n} \xi_{n i}, \max _{1 \leq i \leq n} \eta_{n i}\right) .
$$

For fixed $x, y \in \mathbb{R},[16]$ showed that

$$
\begin{aligned}
& \lim _{n \rightarrow \infty} \mathbb{P}\left(M_{n 1} \leq b_{n}+\frac{x}{b_{n}}, M_{n 2} \leq b_{n}+\frac{y}{b_{n}}\right) \\
= & \lim _{n \rightarrow \infty} F^{n}\left(b_{n}+\frac{x}{b_{n}}, b_{n}+\frac{y}{b_{n}}\right) \\
= & H_{\lambda}(x, y)
\end{aligned}
$$

if $\rho_{n}$ satisfies the following Hüsler-Reiss condition (which is also the necessary condition, see Lemma 21 in [17])

$$
\lim _{n \rightarrow \infty} b_{n}^{2}\left(1-\rho_{n}\right)=2 \lambda^{2} \quad \text { with } \lambda \in[0, \infty],
$$

*Corresponding author.

where the norming constant $b_{n}$ satisfies

$$
\sqrt{2 \pi} n^{-1} b_{n} \exp \left(\frac{b_{n}^{2}}{2}\right)=1
$$

and $H_{\lambda}(x, y)$, the Hüsler-Reiss max-stable distribution, is given by

$$
\begin{aligned}
H_{\lambda}(x, y)= & \exp \left(-\Phi\left(\lambda+\frac{x-y}{2 \lambda}\right) e^{-y}\right. \\
& \left.-\Phi\left(\lambda+\frac{y-x}{2 \lambda}\right) e^{-x}\right),
\end{aligned}
$$

with $\Phi(x)$ denoting the standard Gaussian distribution. Note that from the discussion in [16],

$$
H_{0}(x, y)=\lim _{\lambda \downarrow 0} H_{\lambda}(x, y)=\Lambda(\min (x, y))
$$

and

$$
H_{\infty}(x, y)=\lim _{\lambda \uparrow \infty} H_{\lambda}(x, y)=\Lambda(x) \Lambda(y),
$$

where $\Lambda(x)=\exp \left(-e^{-x}\right), x \in \mathbb{R}$, the standard Gumbel distribution function. We say that $\left\{\xi_{n i}, 1 \leq i \leq n, n \geq 1\right\}$ and $\left\{\eta_{n i}, 1 \leq i \leq n, n \geq 1\right\}$ are asymptotic complete dependent and independent if (1.1) holds with $H_{0}(x, y)$ and $H_{\infty}(x, y)$, respectively.

Motivated by the seminal work of [16], numerous contributions on limiting distributions of extremes of bivariate triangular arrays have appeared in the literature. [15] derived general results for asymptotic dependence structures of bivariate maxima in a triangular array of independent random vectors. [14] considered the maxima of independent and identically distributed bivariate Gaussian random vectors with respect to two arbitrary directions. $[9,10]$ extended the results to the case of triangular arrays of independent elliptical random vectors. Related results can be found in $[4,11,12]$. For statistical applications of Hüsler-Reiss distributions, see [5].

In this paper, we are interested in the uniform convergence rate of bivariate maxima $\mathbf{M}_{n}$ to its ultimate HüslerReiss max-stable distribution. For the univariate case, [3] considered the uniform convergence rate of maxima to its extreme value distribution by imposing some second order regular variation conditions. For the extreme value distributions of given distributions and their associated uniform 
convergence rates, we refer to $[7,8,19,21]$ and references therein. There are relatively few studies on the convergence rates of extremes under multivariate settings. [2] considered the convergence rates of bivariate extreme order statistics under second-order regular varying conditions. For bivariate Hüsler-Reiss Gaussian sequences, recently [6] considered the penultimate and ultimate convergence rate of $\left(n\left(\max _{1 \leq i \leq n} \Phi\left(\xi_{n i}\right)-1\right), n\left(\max _{1 \leq i \leq n} \Phi\left(\eta_{n i}\right)-1\right)\right)$, and [13] derived the second order expansions of the distribution of normalized $\mathbf{M}_{n}$ under the following second order HüslerReiss condition

$$
\lim _{n \rightarrow \infty} b_{n}^{2}\left(\lambda_{n}-\lambda\right)=\alpha \in \mathbb{R}
$$

since $b_{n}^{2} \sim 2 \log n$ as $n \rightarrow \infty$ due to

$$
b_{n}=(2 \log n)^{1 / 2}-\frac{\log \log n+\log 4 \pi}{2(2 \log n)^{1 / 2}}+o\left(\frac{1}{(\log n)^{1 / 2}}\right)
$$

by (1.3), see, e.g., [18, 22].

(ii). Let $\delta_{n}=\left(\lambda_{n}-\lambda\right)^{-1}$. If (1.5) does not converge but $\left|\delta_{n}\right|$ and $b_{n}^{2}$ are the same order, then (2.1) also holds. Proofs are similar, and details are omitted here.

(iii). If $\lim _{n \rightarrow \infty} b_{n}^{2} /\left|\delta_{n}\right|=\infty$, with arguments similar to that of Theorem 1, we can show that

with $\lambda_{n}=\left(\frac{1}{2} b_{n}^{2}\left(1-\rho_{n}\right)\right)^{1 / 2}$ and $\lambda \in(0, \infty)$. So far, there are no results in the literature concerning the uniform convergence rate of the distribution of normalized $\mathbf{M}_{n}$ to its ultimate extreme value distribution. The main goal of this paper is to derive such a result, filling the gap in the current literature. Our proofs show that, for the Hüsler-Reiss Gaussian triangular array, establishing the uniform convergence rate is more technical and complicated than the higher-order expansions of distribution of normalized $\mathbf{M}_{n}$.

The rest of this paper is organized as follows. In Section 2 , we provide the main results, and all proofs are given in Section 3. Auxiliary lemmas and their proofs are deferred to Appendix A.

\section{MAIN RESULTS}

In this section, we provide the main results which show that the uniform convergence rate of $F^{n}\left(b_{n}+x / b_{n}, b_{n}+\right.$ $\left.y / b_{n}\right)$ to its ultimate Hüsler-Reiss max-stable distribution is of order $O(1 / \log n)$. For notational simplicity, let

$$
\Delta\left(F^{n}, H_{\lambda} ; x, y\right)=F^{n}\left(b_{n}+x / b_{n}, b_{n}+y / b_{n}\right)-H_{\lambda}(x, y) .
$$

For the case of $\lambda \in(0, \infty)$, the following theorem establishes the uniform convergence rate under the second-order HüslerReiss condition (1.5).

Theorem 1. For the triangular array of bivariate Gaussian random vectors with each vector following distribution $F$, assume that the second order Hüsler-Reiss condition (1.5) holds with $\lambda_{n}=\left(\frac{1}{2} b_{n}^{2}\left(1-\rho_{n}\right)\right)^{1 / 2}$ and $\lambda \in(0, \infty)$. Then there exist absolute constants $0<D_{1}<D_{2}$ such that

$$
\frac{D_{1}}{\log n}<\sup _{(x, y) \in \mathbb{R}^{2}}\left|\Delta\left(F^{n}, H_{\lambda} ; x, y\right)\right|<\frac{D_{2}}{\log n}
$$

for $n \geq 2$.

Remark 1. (i). Condition (1.5) is equivalent to

$$
\lim _{n \rightarrow \infty}(\log n)\left(\lambda_{n}-\lambda\right)=\alpha / 2
$$

for $n \geq 1$.

(ii). The situation that $\lim _{n \rightarrow \infty} b_{n}^{2} /\left|\delta_{n}\right|=\infty$ is the one that we are not so interested in since (2.2) shows that the convergence rate $1 /\left|\delta_{n}\right|$ is related to correlation $\rho_{n}$ and parameter $\lambda$.

Theorem 1 and the following remark show that the rate of convergence with norming constant $b_{n}$ given by (1.3) is optimal comparing with that with norming constant $\beta_{n}$ denoted by $(2.3)$ even though $b_{n}-\beta_{n}=o\left(1 /(\log n)^{1 / 2}\right)$ by Remark 1.

Remark 3. (i). Assume that the triangular array of bivariate Gaussian random vectors satisfies the second-order Hüsler-Reiss condition (1.5) with $\alpha \in \mathbb{R}$. If the norming constant $b_{n}$ is replaced by $\beta_{n}$ given by

$$
\beta_{n}=2(\log n)^{1 / 2}-\frac{\log \log n+\log 4 \pi}{2(2 \log n)^{1 / 2}},
$$


we can prove that

$$
\begin{aligned}
& \widetilde{\Delta}\left(F^{n}, H_{\lambda} ; x, y\right) \\
= & F^{n}\left(\beta_{n}+x / \beta_{n}, \beta_{n}+y / \beta_{n}\right)-H_{\lambda}(x, y) \\
\sim & {\left[\Phi\left(\lambda+\frac{x-y}{2 \lambda}\right) e^{-y}+\Phi\left(\lambda+\frac{y-x}{2 \lambda}\right) e^{-x}\right] } \\
& \times \frac{(\log \log n)^{2}}{16 \log n} H_{\lambda}(x, y)
\end{aligned}
$$

as $n \rightarrow \infty$ for all $x, y \in \mathbb{R}$, from which shows that the convergence rate is no better than $(\log \log n)^{2} /(16 \log n)$.

(ii). Under the second-order Hüsler-Reiss condition (1.5) with $\alpha= \pm \infty$, we have

$$
\begin{aligned}
& \widetilde{\Delta}\left(F^{n}, H_{\lambda} ; x, y\right) \\
= & {\left[\frac { ( \operatorname { l o g } \operatorname { l o g } n ) ^ { 2 } } { 1 6 \operatorname { l o g } n } \left(\Phi\left(\lambda+\frac{x-y}{2 \lambda}\right) e^{-y}\right.\right.} \\
& \left.+\Phi\left(\lambda+\frac{y-x}{2 \lambda}\right) e^{-x}\right)(1+o(1)) \\
& \left.-\left(\lambda_{n}-\lambda\right) 2 e^{-x} \varphi\left(\lambda+\frac{x-y}{2 \lambda}\right)(1+o(1))\right] H_{\lambda}(x, y)
\end{aligned}
$$

as $n \rightarrow \infty$ for all $x, y \in \mathbb{R}$, where the norming constant $\beta_{n}$ is given by (2.3). By (2.5), we can see that the convergence rate is no better than $\max \left\{(\log \log n)^{2} /(16 \log n),\left|\lambda_{n}-\lambda\right|\right\}$.

For the two extreme cases $\lambda=0$ and $\lambda=\infty$, we need to deal with them separately. For the case of $\lambda=\infty$, the results are stated as follows.

Theorem 2. Let norming constant $b_{n}$ be given by (1.3). For $\rho_{n} \in[-1,1)$,

(i). assertion (2.1) holds if $\rho_{n} \in[-1,0]$.

(ii). if $\rho_{n} \in(0,1)$, assume that (1.2) holds with $\lambda=\infty$ and $\left(\log b_{n}\right) /\left(\left(1-\rho_{n}\right) b_{n}^{2}\right) \rightarrow 0$ as $n \rightarrow \infty$, then (2.1) also holds.

For the case of $\lambda=0$, we have the following results.

Theorem 3. Let norming constant $b_{n}$ be given by (1.3) For $\rho_{n} \in(0,1]$,

(i). assertion (2.1) holds if $\rho_{n} \equiv 1$ for all large $n$.

(ii). if $\rho_{n} \in(0,1)$, assume that $b_{n}^{10}\left(1-\rho_{n}\right) \rightarrow c \in[0, \infty)$ as $n \rightarrow \infty$, then (2.1) also holds.

Remark 4. For the case of $\rho_{n} \in(0,1)$, the proofs of Theorem 2 and Theorem 3 depend heavily on Berman's inequality. In order to derive the upper bound based on Berman's inequality, some sufficient conditions are needed. The condition in Theorem 2 requires that $\left(1-\rho_{n}\right) b_{n}^{2}$ converges to infinity faster than $\log b_{n}$; The condition imposed on Theorem 3(ii) implies that (1.2) holds with $\lambda=0$.

\section{PROOFS}

The aim of this section is to prove our main results. In the sequel, we rewrite $H_{\lambda}(x, y)$ as

$$
H_{\lambda}(x, y)=\exp \left(-e^{-x}-\int_{y}^{\infty} \Phi\left(\lambda+\frac{x-z}{2 \lambda}\right) e^{-z} d z\right) .
$$

For notational simplicity, throughout this paper let

$$
\begin{gathered}
u_{n}(z)=b_{n}+z / b_{n}, \quad z \in \mathbb{R}, \\
A_{n}=b_{n}^{2}\left(\lambda_{n}\left(1-\frac{\lambda_{n}^{2}}{b_{n}^{2}}\right)^{-\frac{1}{2}}-\lambda\right), \\
B_{n}=\frac{1}{2} b_{n}^{2}\left(\frac{1}{\lambda_{n}}\left(1-\frac{\lambda_{n}^{2}}{b_{n}^{2}}\right)^{-\frac{1}{2}}-\frac{1}{\lambda}\right)
\end{gathered}
$$

and

$$
C_{n}=\lambda_{n}\left(1-\frac{\lambda_{n}^{2}}{b_{n}^{2}}\right)^{-\frac{1}{2}}
$$

where the norming constant $b_{n}$ is given by (1.3) and $\lambda_{n}=$ $\left(b_{n}^{2}\left(1-\rho_{n}\right) / 2\right)^{1 / 2}$. If the second-order Hüsler-Reiss condition (1.5) holds with $\lambda \in(0, \infty)$, it is easy to check that

$$
A_{n} \rightarrow \frac{1}{2} \lambda^{3}+\alpha, \quad B_{n} \rightarrow-\frac{1}{2} \alpha \lambda^{-2}+\frac{1}{4} \lambda, \quad C_{n} \rightarrow \lambda
$$

as $n \rightarrow \infty$.

Proof of Theorem 1. By Lemma 1 and Lemma 3 in Appendix A and $b_{n}^{2} \sim 2 \log n$ as $n \rightarrow \infty$, for fixed $x, y \in \mathbb{R}$ we have

$$
\begin{aligned}
\Delta\left(F^{n}, H_{\lambda} ; x, y\right) \sim & \left(e^{-x}\left(1+x+\frac{1}{2} x^{2}\right)\right. \\
& \left.+\frac{1}{2} \kappa_{3}(x, y)+\kappa_{1}(x, y)\right) \\
& \times(2 \log n)^{-1} H_{\lambda}(x, y)
\end{aligned}
$$

as $n \rightarrow \infty$, where $\kappa_{1}(x, y)$ and $\kappa_{3}(x, y)$ respectively are given by (A.4) and (A.13) in Appendix A. Hence there exists an absolute constant $D_{1}>0$ such that

$$
\sup _{(x, y) \in \mathbb{R}^{2}}\left|\Delta\left(F^{n}, H_{\lambda} ; x, y\right)\right| \geq \frac{D_{1}}{\log n}
$$

for $n \geq 2$. Thus we need to show further that

$$
\sup _{(x, y) \in \mathbb{R}^{2}}\left|\Delta\left(F^{n}, H_{\lambda} ; x, y\right)\right| \leq \frac{D_{2}}{\log n}
$$

for $n \geq 2$, where $D_{2}$ is an absolute constant. By Lemma 4 in Appendix A, it suffices to prove the following inequalities:

$$
\sup _{(x, y) \in\left[-c_{n}, d_{n}\right] \times\left[-c_{n}, d_{n}\right]}\left|\Delta\left(F^{n}, H_{\lambda} ; x, y\right)\right| \leq \mathbb{D}_{2} b_{n}^{-2},
$$




$$
\begin{aligned}
& \sup _{(x, y) \in\left[-c_{n}, d_{n}\right] \times\left[d_{n}, \infty\right)}\left|\Delta\left(F^{n}, H_{\lambda} ; x, y\right)\right| \leq \mathbb{D}_{3} b_{n}^{-2}, \\
& \sup _{(x, y) \in\left[d_{n}, \infty\right) \times\left[d_{n}, \infty\right)}\left|\Delta\left(F^{n}, H_{\lambda} ; x, y\right)\right| \leq \mathbb{D}_{4} b_{n}^{-2}
\end{aligned}
$$

for $n \geq n_{0}$ since both

$$
\sup _{(x, y) \in \mathbb{R} \times\left(-\infty,-c_{n}\right]}\left|\Delta\left(F^{n}, H_{\lambda} ; x, y\right)\right| \leq \mathbb{D}_{1} b_{n}^{-2}
$$

and

$$
\sup _{(x, y) \in\left[d_{n}, \infty\right) \times\left[-c_{n}, d_{n}\right]}\left|\Delta\left(F^{n}, H_{\lambda} ; x, y\right)\right| \leq \mathbb{D}_{3} b_{n}^{-2}
$$

also hold by the arguments similar to those used in (A.20) and (3.5), where $\mathbb{D}_{i}>0,2 \leq i \leq 4$, are absolute constants, and $c_{n}$ and $d_{n}$ are given by Lemma 2 in Appendix A, i.e.,

$$
c_{n}=\log \log b_{n}>0, \quad d_{n}=-\log \log \frac{b_{n}^{2}}{b_{n}^{2}-1}>0
$$

for $n \geq n_{0}$. Note that $x \geq-c_{n}$ implies

$$
u_{n}(x) \geq b_{n}-\frac{c_{n}}{b_{n}}=b_{n}\left(1-\frac{\log \log b_{n}^{2}}{b_{n}^{2}}\right)>0, \quad n \geq n_{0} .
$$

So, the desired upper bound (3.3) can be obtained by (3.4)(3.8) and (A.20).

For the rest of the proof, let $\mathbb{C}_{i}, 7 \leq i \leq 13$, stand for absolute positive constants.

For $(x, y) \in\left[-c_{n}, \infty\right) \times\left[-c_{n}, \infty\right)$, let $\psi_{n}(x, y)=1-$ $F\left(u_{n}(x), u_{n}(y)\right)$, then

$$
n \log F\left(u_{n}(x), u_{n}(y)\right)=-n \psi_{n}(x, y)-R_{n}(x, y),
$$

where

$$
0<R_{n}(x, y)<\frac{n \psi_{n}^{2}(x, y)}{2\left(1-\psi_{n}(x, y)\right)}
$$

due to

$$
-z-\frac{z^{2}}{2(1-z)}<\log (1-z)<-z, \quad 0<z<1 .
$$

By (1.3), (A.17) and (A.19),

$$
\begin{aligned}
& \sup _{(x, y) \in\left[-c_{n}, \infty\right) \times\left[-c_{n}, \infty\right)} \psi_{n}(x, y) \\
\leq & 1-F\left(u_{n}\left(-c_{n}\right), u_{n}\left(-c_{n}\right)\right) \\
= & n^{-1} \int_{-c_{n}}^{\infty} \Phi\left(\frac{u_{n}\left(-c_{n}\right)-\rho_{n} u_{n}(z)}{\sqrt{1-\rho_{n}^{2}}}\right) e^{-z} \exp \left(-\frac{z^{2}}{2 b_{n}^{2}}\right) d z \\
& +1-\Phi\left(u_{n}\left(-c_{n}\right)\right) \\
\leq & \frac{1}{\sqrt{2 \pi}}\left(b_{n}-\frac{c_{n}}{b_{n}}\right)^{-1} \exp \left(-\frac{\left(b_{n}-\frac{c_{n}}{b_{n}}\right)^{2}}{2}\right)+n^{-1} \int_{-c_{n}}^{\infty} e^{-z} d z \\
\leq & n^{-1}\left(\log b_{n}^{2}\right)\left(1+\left(1-b_{n}^{-2} \log \log b_{n}^{2}\right)^{-1}\right) \\
< & \mathbb{C}_{7}<1
\end{aligned}
$$

for $n \geq n_{0}$, which implies

$$
\begin{aligned}
0 & <\sup _{(x, y) \in\left[-c_{n}, \infty\right) \times\left[-c_{n}, \infty\right)} R_{n}(x, y) \\
& <\sup _{(x, y) \in\left[-c_{n}, \infty\right) \times\left[-c_{n}, \infty\right)} \frac{n \psi_{n}^{2}(x, y)}{2\left(1-\psi_{n}(x, y)\right)} \\
& <b_{n}^{-2}
\end{aligned}
$$

for $n \geq n_{0}$. Hence by $e^{x} \geq 1+x, x \in \mathbb{R}$ we have

$$
1-\exp \left(-R_{n}(x, y)\right) \leq R_{n}(x, y)<b_{n}^{-2}
$$

for $n \geq n_{0}$. Hence by (3.11) we have

$$
\begin{aligned}
\left|\Delta\left(F^{n}, H_{\lambda} ; x, y\right)\right|< & H_{\lambda}(x, y) N_{n}(x, y)\left|Q_{n}(x, y)-1\right| \\
& +H_{\lambda}(x, y)\left|N_{n}(x, y)-1\right| \\
< & H_{\lambda}(x, y)\left|Q_{n}(x, y)-1\right|+b_{n}^{-2}
\end{aligned}
$$

for $n>n_{0}$, where

$$
\begin{gathered}
Q_{n}(x, y)=\exp \left(-n \psi_{n}(x, y)+\Phi\left(\lambda+\frac{x-y}{2 \lambda}\right) e^{-y}\right. \\
\left.+\Phi\left(\lambda+\frac{y-x}{2 \lambda}\right) e^{-x}\right)
\end{gathered}
$$

and

$$
N_{n}(x, y)=\exp \left(-R_{n}(x, y)\right)
$$

Note that

$$
-n\left(1-\Phi\left(u_{n}(x)\right)\right)+e^{-x}=\left(1+b_{n}^{-2} x\right)^{-1} e^{-x} Z_{n}(x)
$$

with

$Z_{n}(x)=-\exp \left(-\frac{x^{2}}{2 b_{n}^{2}}\right)\left(1-\theta_{n} b_{n}^{-2}\left(1+b_{n}^{-2} x\right)^{-2}\right)+1+b_{n}^{-2} x$,

where $0<\theta_{n}<1$, cf., [7]. By arguments similar to those used in [7], we have

$$
b_{n}^{-2} x<Z_{n}(x)<b_{n}^{-2}\left(2^{-1} x^{2}+\left(1+b_{n}^{-2} x\right)^{-2}+x\right)
$$

by $1-z<e^{-z}<1$ for $z>0$, which implies

$$
\left|Z_{n}(x)\right|<b_{n}^{-2}\left(2^{-1} x^{2}+\left(1+b_{n}^{-2} x\right)^{-2}+|x|\right) .
$$

Combining with (3.13), we have

$$
\left|-n\left(1-\Phi\left(u_{n}(x)\right)\right)+e^{-x}\right|<\mathbb{C}_{8} b_{n}^{-2} e^{-x}\left(\frac{x^{2}}{2}+|x|+\mathbb{C}_{9}\right)
$$

for $n \geq n_{0}$, if $x \geq-c_{n}$.

Similarly, for $x \geq-c_{n}$ we have

$$
\mid-\int_{y}^{\infty} \Phi\left(\frac{u_{n}(x)-\rho_{n} u_{n}(z)}{\sqrt{1-\rho_{n}^{2}}}\right) e^{-z} \exp \left(-\frac{z^{2}}{2 b_{n}^{2}}\right) d z
$$




$$
\begin{aligned}
& +\int_{y}^{\infty} \Phi\left(\lambda+\frac{x-z}{2 \lambda}\right) e^{-z} d z \mid \\
< & \int_{y}^{\infty}\left|\Phi\left(\frac{u_{n}(x)-\rho_{n} u_{n}(z)}{\sqrt{1-\rho_{n}^{2}}}\right)-\Phi\left(\lambda+\frac{x-z}{2 \lambda}\right)\right| e^{-z} d z \\
& +b_{n}^{-2} e^{-y}\left(\frac{y^{2}}{2}+y+1\right)
\end{aligned}
$$

by using $\left|e^{-x}-1\right|<x$ for $x>0$.

First, we prove (3.4). Combining (A.9), (3.14) and (3.15), we have

$$
\begin{aligned}
& \mid-n \psi_{n}(x, y)+\Phi\left(\lambda+\frac{x-y}{2 \lambda}\right) e^{-y}+\Phi\left(\lambda+\frac{y-x}{2 \lambda}\right) e^{-x} \\
& \leq\left|-n\left(1-\Phi\left(u_{n}(x)\right)\right)+e^{-x}\right|+\mid \int_{y}^{\infty} \Phi\left(\lambda+\frac{x-z}{2 \lambda}\right) e^{-z} d z \\
& -\int_{y}^{\infty} \Phi\left(\frac{u_{n}(x)-\rho_{n} u_{n}(z)}{\sqrt{1-\rho_{n}^{2}}}\right) e^{-z} \exp \left(-\frac{z^{2}}{2 b_{n}^{2}}\right) d z \mid \\
& \leq \mathbb{C}_{8} b_{n}^{-2} e^{-x}\left(\frac{x^{2}}{2}+|x|+\mathbb{C}_{9}\right)+b_{n}^{-2} e^{-y}\left(\frac{y^{2}}{2}+y+1\right) \\
& +b_{n}^{-2}\left(e^{-y}\left(\mathbb{C}_{1}|y|+\mathbb{C}_{2}\right)+\mathbb{C}_{3} e^{-x}|x|+\mathbb{C}_{4}\right) \\
& \leq b_{n}^{-2}\left[\mathbb{C}_{8} e^{-x}\left(\frac{x^{2}}{2}+\left(1+\frac{\mathbb{C}_{3}}{\mathbb{C}_{8}}\right)|x|+\mathbb{C}_{9}\right)\right. \\
& \left.+e^{-y}\left(\frac{y^{2}}{2}+\left(\mathbb{C}_{1}+1\right)|y|+\mathbb{C}_{2}+1\right)+\mathbb{C}_{4}\right]
\end{aligned}
$$

for $n \geq n_{0}$. Note that

$$
e^{-x} x^{2} \leq 4, \quad e^{-x} x \leq 1, \quad \text { for } x>0
$$

and by using (3.16) we have

$$
\begin{aligned}
& \quad\left|-n \psi_{n}(x, y)+\Phi\left(\lambda+\frac{x-y}{2 \lambda}\right) e^{-y}+\Phi\left(\lambda+\frac{y-x}{2 \lambda}\right) e^{-x}\right| \\
& \leq \mathbb{C}_{10} b_{n}^{-2} \leq 1
\end{aligned}
$$

for $n \geq n_{0}$ and any $(x, y) \in\left[0, d_{n}\right] \times\left[0, d_{n}\right]$. Hence for $(x, y) \in$ $\left[0, d_{n}\right] \times\left[0, d_{n}\right]$

$$
\begin{aligned}
& H_{\lambda}(x, y)\left|Q_{n}(x, y)-1\right| \\
\leq & H_{\lambda}(x, y) \mid-n \psi_{n}(x, y)+\Phi\left(\lambda+\frac{x-y}{2 \lambda}\right) e^{-y} \\
& +\Phi\left(\lambda+\frac{y-x}{2 \lambda}\right) e^{-x} \mid\left(1+\exp \mid-n \psi_{n}(x, y)\right. \\
& \left.+\Phi\left(\lambda+\frac{x-y}{2 \lambda}\right) e^{-y}+\Phi\left(\lambda+\frac{y-x}{2 \lambda}\right) e^{-x} \mid\right) \\
\leq & \mathbb{C}_{10}(e+1) b_{n}^{-2}
\end{aligned}
$$

since $\left|e^{x}-1\right| \leq|x|\left(e^{|x|}+1\right), x \in \mathbb{R}$.
For the case of $(x, y) \in\left[-c_{n}, 0\right] \times\left[-c_{n}, 0\right],(3.16)$ implies

$\left|-n \psi_{n}(x, y)+\Phi\left(\lambda+\frac{x-y}{2 \lambda}\right) e^{-y}+\Phi\left(\lambda+\frac{y-x}{2 \lambda}\right) e^{-x}\right| \leq 1$

for $n \geq n_{0}$. For $(x, y) \in\left[-c_{n}, 0\right] \times\left[-c_{n}, 0\right]$,

$$
\begin{aligned}
\leq & (e+1) b_{n}^{-2}\left[\mathbb{C}_{8} e^{-1-\frac{x^{2}}{2}}\left(\frac{x^{2}}{2}+\left(1+\frac{\mathbb{C}_{3}}{\mathbb{C}_{8}}\right)|x|+\mathbb{C}_{9}\right)\right. \\
& \left.+e^{-1-\frac{y^{2}}{2}}\left(\frac{y^{2}}{2}+\left(\mathbb{C}_{1}+1\right) y+\mathbb{C}_{2}+1\right)+\mathbb{C}_{4}\right] \\
\leq & \mathbb{C}_{10}(e+1) b_{n}^{-2}
\end{aligned}
$$

for $n \geq n_{0}$ by noting that $e^{-t}>1-t+\frac{t^{2}}{2}$ for $t<0$, and $\frac{t^{2}}{2} \exp \left(-\frac{t^{2}}{2}\right) \leq 1$ and $t \exp \left(-\frac{t^{2}}{2}\right) \leq 1$ for $t>0$.

By arguments similar to those used in (3.18) and (3.19), for $n \geq n_{0}$ we have

$$
H_{\lambda}(x, y)\left|Q_{n}(x, y)-1\right| \leq \mathbb{C}_{10}(e+1) b_{n}^{-2}
$$

if $(x, y) \in\left[0, d_{n}\right] \times\left[-c_{n}, 0\right]$ or $(x, y) \in\left[-c_{n}, 0\right] \times\left[0, d_{n}\right]$.

Combining (3.18)-(3.20) and (3.12), we get

$$
\sup _{(x, y) \in\left[-c_{n}, d_{n}\right] \times\left[-c_{n}, d_{n}\right]}\left|F^{n}\left(u_{n}(x), u_{n}(y)\right)-H_{\lambda}(x, y)\right| \leq \mathbb{D}_{2} b_{n}^{-2},
$$

which completes the proof of (3.4).

Second, we consider the case in which $(x, y) \in\left[-c_{n}, d_{n}\right] \times$ $\left[d_{n}, \infty\right)$. By $\left(\right.$ A.10), (3.14) and (3.15), for all $y \in\left[d_{n}, \infty\right)$ we have

$$
\begin{aligned}
& \left|-n \psi_{n}(x, y)+\Phi\left(\lambda+\frac{x-y}{2 \lambda}\right) e^{-y}+\Phi\left(\lambda+\frac{y-x}{2 \lambda}\right) e^{-x}\right| \\
\leq & b_{n}^{-2}\left(\mathbb{C}_{8} e^{-x}\left(\frac{x^{2}}{2}+|x|+\mathbb{C}_{9}\right)+\left(\mathbb{C}_{5}+4\right)\right)
\end{aligned}
$$

for $n \geq n_{0}$. Thus by the arguments similar to those used in (3.18) and (3.19), we have

$$
H_{\lambda}(x, y)\left|Q_{n}(x, y)-1\right| \leq \mathbb{C}_{11} b_{n}^{-2}
$$

for $n \geq n_{0}$ if $(x, y) \in\left[0, d_{n}\right] \times\left[d_{n}, \infty\right)$, and

$$
H_{\lambda}(x, y)\left|Q_{n}(x, y)-1\right| \leq \mathbb{C}_{12} b_{n}^{-2}
$$

for $n \geq n_{0}$ if $(x, y) \in\left[-c_{n}, 0\right] \times\left[d_{n}, \infty\right)$. Combining (3.12), (3.21) and (3.22), we can get (3.5).

Finally, we prove (3.6). Note that $d_{n}=-\log \log \frac{b_{n}^{2}}{b_{n}^{2}-1}$, we have

$$
\begin{aligned}
& \sup _{(x, y) \in\left[d_{n}, \infty\right) \times\left[d_{n}, \infty\right)}\left(1-H_{\lambda}(x, y)\right) \\
\leq & 1-H_{\lambda}\left(d_{n}, d_{n}\right) \\
\leq & b_{n}^{-2}+1-\exp \left(-\int_{d_{n}}^{\infty} e^{-z} d z\right)=2 b_{n}^{-2} .
\end{aligned}
$$


By using (3.11) and $e^{z}>1+z, z \in \mathbb{R}$, we have

$$
\begin{aligned}
& \sup _{(x, y) \in\left[d_{n}, \infty\right) \times\left[d_{n}, \infty\right)}\left(1-F^{n}\left(u_{n}(x), u_{n}(y)\right)\right) \\
\leq & n \psi_{n}\left(d_{n}, d_{n}\right)+R_{n}\left(d_{n}, d_{n}\right) \\
\leq & \left(1+\mathbb{C}_{13}\right) b_{n}^{-2},
\end{aligned}
$$

where the last inequality is due to

$$
\begin{aligned}
& n \psi_{n}\left(d_{n}, d_{n}\right) \\
= & \int_{d_{n}}^{\infty} \Phi\left(\frac{u_{n}\left(d_{n}\right)-\rho_{n} u_{n}(z)}{\sqrt{1-\rho_{n}^{2}}}\right) e^{-z} \exp \left(-\frac{z^{2}}{2 b_{n}^{2}}\right) d z \\
& +n\left(1-\Phi\left(u_{n}\left(d_{n}\right)\right)\right) \\
< & n\left(b_{n}+\frac{d_{n}}{b_{n}}\right)^{-1} \frac{1}{\sqrt{2 \pi}} \exp \left(-\frac{\left(b_{n}+\frac{d_{n}}{b_{n}}\right)^{2}}{2}\right)+\int_{d_{n}}^{\infty} e^{-z} d z \\
= & \left(1+\frac{d_{n}}{b_{n}^{2}}\right)^{-1} e^{-d_{n}} \exp \left(-\frac{d_{n}^{2}}{2 b_{n}^{2}}\right)+e^{-d_{n}} \\
< & \mathbb{C}_{13} b_{n}^{-2}
\end{aligned}
$$

for $n \geq n_{0}$ by using (A.17), (A.19) and

$$
\begin{aligned}
b_{n}^{2} e^{-d_{n}} & =b_{n}^{2}\left(-\log \left(1-b_{n}^{-2}\right)\right) \\
& <b_{n}^{2}\left(b_{n}^{-2}+\frac{b_{n}^{-4}}{2\left(1-b_{n}^{-2}\right)}\right) \\
& =1+\frac{1}{2\left(b_{n}^{2}-1\right)}
\end{aligned}
$$

as $-z-\frac{z^{2}}{2(1-z)}<\log (1-z), 0<z<1$.

Combining (3.23) with (3.24), we have

$$
\sup _{(x, y) \in\left[d_{n}, \infty\right) \times\left[d_{n}, \infty\right)}\left|\Delta\left(F^{n}, H_{\lambda} ; x, y\right)\right| \leq \mathbb{D}_{4} b_{n}^{-2}
$$

for $n \geq n_{0}$, which completes the proof of (3.6). The proof of Theorem 1 is complete.

Proof of Theorem 2. (i). For the case of $\rho_{n} \in[-1,0]$, we first consider two special cases, $\rho_{n} \equiv-1$ and $\rho_{n} \equiv 0$ respectively, then extend the result to the general case $\rho_{n} \in$ $[-1,0]$ by using Slepian's Lemma.

Note that, for $\rho_{n} \equiv-1$ and $\rho_{n} \equiv 0$, for the upper bound of (2.1), by Lemma 4 in Appendix A we only need to check that

$$
\sup _{(x, y) \in\left[-c_{n}, \infty\right) \times\left[-c_{n}, \infty\right)}\left|\Delta\left(F^{n}, H_{\infty} ; x, y\right)\right|<\mathbb{D}_{5} b_{n}^{-2}
$$

for large $n$, where $c_{n}=\log \log b_{n}^{2}$, and $\mathbb{D}_{5}$ is an absolute positive constant.

Let $(\xi, \eta)$ be a bivariate Gaussian random vector with correlation $\rho_{n} \equiv 0$. By (A.17) and (A.19), for $(x, y) \in$ $\left[-c_{n}, \infty\right) \times\left[-c_{n}, \infty\right)$ we have

$$
n \mathbb{P}\left(\xi>u_{n}(x), \eta>u_{n}(y)\right)
$$

$$
\begin{aligned}
& <\frac{1}{\sqrt{2 \pi} b_{n} \exp \left(\frac{b_{n}^{2}}{2}\right)}\left(1+\frac{x}{b_{n}^{2}}\right)^{-1}\left(1+\frac{y}{b_{n}^{2}}\right)^{-1} e^{-x-y} \\
& <b_{n}^{-2} \frac{b_{n}\left(\log b_{n}^{2}\right)^{2}\left(1-\frac{\log \log b_{n}^{2}}{b_{n}^{2}}\right)^{-2}}{\sqrt{2 \pi} \exp \left(\frac{b_{n}^{2}}{2}\right)} \\
& <b_{n}^{-2}
\end{aligned}
$$

for large $n$.

By using (3.26) and (3.14) and arguments similar to those used in [7], for $(x, y) \in\left[-c_{n}, \infty\right) \times\left[-c_{n}, \infty\right)$ we have

$$
\begin{aligned}
\mid & n\left(1-F\left(u_{n}(x), u_{n}(y)\right)\right)+e^{-x}+e^{-y} \mid \\
\leq & \left|-n\left(1-\Phi\left(u_{n}(x)\right)\right)+e^{-x}\right|+\left|-n\left(1-\Phi\left(u_{n}(y)\right)\right)+e^{-y}\right| \\
& +n \mathbb{P}\left(\xi>u_{n}(x), \eta>u_{n}(y)\right) \\
\leq & b_{n}^{-2}\left(1+\mathbb{C}_{8} e^{-x}\left(x^{2} / 2+|x|+\mathbb{C}_{9}\right)\right. \\
& \left.+\mathbb{C}_{8} e^{-y}\left(y^{2} / 2+|y|+\mathbb{C}_{9}\right)\right)
\end{aligned}
$$

for large $n$. Note that, by (3.27) and (3.17), for large $n$ we have

$$
\begin{aligned}
& \sup _{(x, y) \in\left[-c_{n}, 0\right] \times[0, \infty)}\left|-n\left(1-F\left(u_{n}(x), u_{n}(y)\right)\right)+e^{-x}+e^{-y}\right| \\
\leq & b_{n}^{-2}\left(\mathbb{C}_{8}\left(\log b_{n}^{2}\right)\left(\left(\log \log b_{n}^{2}\right)^{2} / 2+\log \log b_{n}^{2}+\mathbb{C}_{9}\right)\right. \\
+ & \left.\mathbb{C}_{8}\left(3+\mathbb{C}_{9}\right)+1\right) \leq 1 .
\end{aligned}
$$

Obviously, for $\rho_{n} \equiv 0$,

$$
\begin{aligned}
& Q_{n}(x, y)=\exp (-n\left(1-\Phi\left(u_{n}(x)\right)\right)+e^{-x}-n\left(1-\Phi\left(u_{n}(y)\right)\right) \\
&\left.+e^{-y}+n \mathbb{P}\left(\xi>u_{n}(x), \eta>u_{n}(y)\right)\right)
\end{aligned}
$$

So, for $(x, y) \in\left[-c_{n}, 0\right) \times[0, \infty)$,

$$
\begin{aligned}
& H_{\infty}(x, y)\left|Q_{n}(x, y)-1\right| \\
\leq & H_{\infty}(x, y)\left|-n\left(1-F\left(u_{n}(x), u_{n}(y)\right)\right)+e^{-x}+e^{-y}\right| \\
& \times\left(\exp \left|-n\left(1-F\left(u_{n}(x), u_{n}(y)\right)\right)+e^{-x}+e^{-y}\right|+1\right) \\
\leq & b_{n}^{-2}(e+1)\left(1+\mathbb{C}_{8}\left(3+\mathbb{C}_{9}\right)\right. \\
& \left.+\mathbb{C}_{8} \exp \left(-e^{-x}-x\right)\left(x^{2} / 2+|x|+\mathbb{C}_{9}\right)\right) \\
\leq & b_{n}^{-2}(e+1)\left(1+\mathbb{C}_{8}\left(3+\mathbb{C}_{9}\right)\right. \\
& \left.+\mathbb{C}_{8} \exp \left(-1-x^{2} / 2\right)\left(x^{2} / 2+|x|+\mathbb{C}_{9}\right)\right) \\
\leq & \left(5 \mathbb{C}_{8}+2 \mathbb{C}_{8} \mathbb{C}_{9}+1\right)(e+1) b_{n}^{-2}
\end{aligned}
$$

for large $n$. Similarly, for large $n$ we have

$$
\begin{aligned}
& \sup _{(x, y) \in[0, \infty) \times\left[-c_{n}, 0\right]} H_{\infty}(x, y)\left|Q_{n}(x, y)-1\right| \\
& \leq\left(5 \mathbb{C}_{8}+2 \mathbb{C}_{8} \mathbb{C}_{9}+1\right)(e+1) b_{n}^{-2},
\end{aligned}
$$




$$
\begin{aligned}
& \sup _{(x, y) \in[0, \infty) \times[0, \infty)} H_{\infty}(x, y)\left|Q_{n}(x, y)-1\right| \\
& \leq\left(6 \mathbb{C}_{8}+2 \mathbb{C}_{8} \mathbb{C}_{9}+1\right)(e+1) b_{n}^{-2}
\end{aligned}
$$

and

$$
\begin{aligned}
& \sup _{(x, y) \in\left[-c_{n}, 0\right] \times\left[-c_{n}, 0\right]} H_{\infty}(x, y)\left|Q_{n}(x, y)-1\right| \\
& \leq\left(4 \mathbb{C}_{8}+2 \mathbb{C}_{8} \mathbb{C}_{9}+1\right)(e+1) b_{n}^{-2} .
\end{aligned}
$$

Combining above with (3.12), we can get (3.25), hence the upper bound in (2.1) is derived. For the lower bound of (2.1) as $\rho_{n} \equiv 0$, by (A.16) and arguments similar to those used in [7], we have

$$
\begin{aligned}
& 1-F\left(u_{n}(x), u_{n}(y)\right) \\
= & n^{-1}\left[e^{-x}+e^{-y}-b_{n}^{-2}\left(e^{-x}\left(x^{2} / 2+x+1\right)\right.\right. \\
& \left.\left.+e^{-y}\left(y^{2} / 2+y+1\right)+O\left(b_{n}^{-2}\right)\right)\right]
\end{aligned}
$$

for large $n$, where $x, y$ are fixed real constants. Hence,

$$
\begin{aligned}
& F^{n}\left(u_{n}(x), u_{n}(y)\right)-H_{\infty}(x, y) \\
= & b_{n}^{-2} H_{\infty}(x, y)\left(e^{-x}\left(x^{2} / 2+x+1\right)\right. \\
& \left.+e^{-y}\left(y^{2} / 2+y+1\right)+O\left(b_{n}^{-2}\right)\right)
\end{aligned}
$$

for large $n$, which implies the left hand side inequality in (2.1). From whence (2.1) is derived for $\rho_{n} \equiv 0$.

Next we consider the case of $\rho_{n} \equiv-1$. For $(x, y) \in$ $\left[-c_{n}, \infty\right) \times\left[-c_{n}, \infty\right)$, noting that for large $n$ we have

$$
\begin{aligned}
Q_{n}(x, y)= & \exp \left(-n\left(1-\Phi\left(u_{n}(x)\right)\right)+e^{-x}\right. \\
& \left.-n\left(1-\Phi\left(u_{n}(y)\right)\right)+e^{-y}\right)
\end{aligned}
$$

as $\mathbb{P}\left(\xi>u_{n}(x), \eta>u_{n}(y)\right)=0$ for large $n$. By arguments similar to that of the case of $\rho_{n} \equiv 0$, we can derive (3.25). Finally the lower bound of (2.1) can be derived by noting that (3.28) also holds if $\rho_{n} \equiv-1$.

Let us now turn to the general case of $\rho_{n} \in[-1,0]$. We just proved that (2.1) holds for $\rho_{n} \equiv-1$ and $\rho_{n} \equiv 0$, respectively. Hence by Slepian's Lemma, one can check that (2.1) also holds if $\rho_{n} \in[-1,0]$.

(ii). For the case of $\rho_{n} \in(0,1)$. If $(x, y) \in\left[-c_{n}, \infty\right) \times$ $\left[-c_{n}, \infty\right)$, by Berman's inequality in [18], we have

$$
\begin{aligned}
& \left|F^{n}\left(u_{n}(x), u_{n}(y)\right)-\left(\Phi\left(u_{n}(x)\right) \Phi\left(u_{n}(y)\right)\right)^{n}\right| \\
\leq & \mathbb{C}_{14} b_{n}^{-2} \exp \left(-\frac{\left(1-\rho_{n}\right) b_{n}^{2}}{4}+3 \log b_{n}+2 \log \log b_{n}^{2}\right) \\
\leq & b_{n}^{-2}
\end{aligned}
$$

for large $n$ due to $\lim _{n \rightarrow \infty}\left(1-\rho_{n}\right)^{-1} b_{n}^{-2} \log b_{n}=0$, where $\mathbb{C}_{14}$ is an absolute positive constant.

From the proof of (A.21), it shows that

$$
\sup _{(x, y) \in\left(-\infty,-c_{n}\right] \times \mathbb{R}} F^{n}\left(u_{n}(x), u_{n}(y)\right)<\mathbb{C}_{6} b_{n}^{-2}
$$

for large $n$ if $\rho_{n} \in[-1,1]$, hence

$$
\begin{aligned}
\sup _{(x, y) \in\left(-\infty,-c_{n}\right] \times \mathbb{R}} & \left|F^{n}\left(u_{n}(x), u_{n}(y)\right)-\left(\Phi\left(u_{n}(x)\right) \Phi\left(u_{n}(y)\right)\right)^{n}\right| \\
& <2 \mathbb{C}_{6} b_{n}^{-2}
\end{aligned}
$$

for large $n$. Similarly,

$$
\begin{aligned}
& \sup _{(x, y) \in \mathbb{R} \times\left(-\infty,-c_{n}\right]}\left|F^{n}\left(u_{n}(x), u_{n}(y)\right)-\left(\Phi\left(u_{n}(x)\right) \Phi\left(u_{n}(y)\right)\right)^{n}\right| \\
&<2 \mathbb{C}_{6} b_{n}^{-2}
\end{aligned}
$$

for large $n$. Combining (3.29), (3.31), (3.32), and (2.1) for the case of $\rho_{n} \equiv 0$, the upper bound of (2.1) is derived if $\rho_{n} \in(0,1)$.

Next we derive the lower bound in (2.1). For fixed $x, y \in$ $\mathbb{R}$, by Mills' ratio we have

$$
1-\Phi\left(\frac{u_{n}(x)-\rho_{n} u_{n}(z)}{\sqrt{1-\rho_{n}^{2}}}\right)<\frac{\exp \left(-\frac{\left(1-\rho_{n}\right) b_{n}^{2}}{4}-\frac{x}{1+\rho_{n}}+\frac{\rho_{n} z}{1+\rho_{n}}\right)}{\sqrt{2 \pi}\left(\lambda_{n}+\frac{x-z}{2 \lambda_{n}}+\frac{\lambda_{n} z}{b_{n}^{2}}\right)}
$$

if $y<z<4 \log b_{n}$. Hence,

$$
\int_{y}^{4 \log b_{n}}\left(1-\Phi\left(\frac{u_{n}(x)-\rho_{n} u_{n}(z)}{\sqrt{1-\rho_{n}^{2}}}\right)\right) e^{-z} d z=O\left(b_{n}^{-4}\right)
$$

for large $n$. Similarly, for large $n$ we have

$$
\int_{y}^{4 \log b_{n}}\left(1-\Phi\left(\frac{u_{n}(x)-\rho_{n} u_{n}(z)}{\sqrt{1-\rho_{n}^{2}}}\right)\right) e^{-z} z^{2} d z=O\left(b_{n}^{-4}\right) .
$$

Note that

$$
\int_{4 \log b_{n}}^{\infty} \Phi\left(\frac{u_{n}(x)-\rho_{n} u_{n}(z)}{\sqrt{1-\rho_{n}^{2}}}\right) e^{-z}\left(1-\frac{z^{2}}{2 b_{n}^{2}}\right) d z=O\left(b_{n}^{-4}\right)
$$

for large $n$. Hence by (3.34) and (3.35), for large $n$ we have

$$
\begin{aligned}
& \int_{y}^{\infty} \Phi\left(\frac{u_{n}(x)-\rho_{n} u_{n}(z)}{\sqrt{1-\rho_{n}^{2}}}\right) e^{-z} \exp \left(-\frac{z^{2}}{2 b_{n}^{2}}\right) d z \\
= & \int_{y}^{4 \log b_{n}}\left(\Phi\left(\frac{u_{n}(x)-\rho_{n} u_{n}(z)}{\sqrt{1-\rho_{n}^{2}}}\right)-1\right) e^{-z} d z \\
& +2^{-1} b_{n}^{-2} \int_{y}^{4 \log b_{n}}\left(1-\Phi\left(\frac{u_{n}(x)-\rho_{n} u_{n}(z)}{\sqrt{1-\rho_{n}^{2}}}\right)\right) e^{-z} z^{2} d z \\
& +\int_{4 \log b_{n}}^{\infty} \Phi\left(\frac{u_{n}(x)-\rho_{n} u_{n}(z)}{\sqrt{1-\rho_{n}^{2}}}\right) e^{-z}\left(1-\frac{z^{2}}{2 b_{n}^{2}}\right) d z \\
& +\int_{y}^{4 \log b_{n}} e^{-z}\left(1-\frac{z^{2}}{2 b_{n}^{2}}\right) d z+O\left(b_{n}^{-4}\right)
\end{aligned}
$$


$=e^{-y}-b_{n}^{-2} e^{-y}\left(\frac{y^{2}}{2}+y+1\right)+O\left(b_{n}^{-4}\right)$.

Combining with (A.16), we have

$$
\begin{aligned}
& 1-F\left(u_{n}(x), u_{n}(y)\right) \\
= & n^{-1} \int_{y}^{\infty} \Phi\left(\frac{u_{n}(x)-\rho_{n} u_{n}(z)}{\sqrt{1-\rho_{n}^{2}}}\right) e^{-z} \exp \left(-\frac{z^{2}}{2 b_{n}^{2}}\right) d z \\
& +1-\Phi\left(u_{n}(x)\right) \\
= & n^{-1}\left[e^{-x}+e^{-y}-b_{n}^{-2}\left(e^{-x}\left(\frac{x^{2}}{2}+x+1\right)\right.\right. \\
& \left.\left.+e^{-y}\left(\frac{y^{2}}{2}+y+1\right)+O\left(b_{n}^{-2}\right)\right)\right]
\end{aligned}
$$

for large $n$, so that the lower bound in (2.1) can be derived if $\rho_{n} \in(0,1)$, which completes the proof.

Proof of Theorem 3. (i). For the case of $\rho_{n} \equiv 1$. Note that

$$
F^{n}\left(u_{n}(x), u_{n}(y)\right)=\Phi^{n}\left(b_{n}+\min (x, y) / b_{n}\right)
$$

and $H_{0}(x, y)=\Lambda(\min (x, y))$. Hence by the arguments provided by [7], we can derive (2.1) if $\rho_{n} \equiv 1$.

(ii). If $\rho_{n} \in(0,1)$ such that $b_{n}^{10}\left(1-\rho_{n}\right) \rightarrow c \in[0, \infty)$ as $n \rightarrow \infty$, this implies that (1.2) holds with $\lambda=0$. First, note that, for $(x, y) \in\left[-c_{n}, \infty\right) \times\left[-c_{n}, \infty\right)$ and large $n$, by Berman's inequality in [20], we have

$$
\begin{aligned}
& \left|F^{n}\left(u_{n}(x), u_{n}(y)\right)-\Phi^{n}\left(b_{n}+\min (x, y) / b_{n}\right)\right| \\
< & \mathbb{C}_{15} n\left(\frac{\pi}{2}-\arcsin \rho_{n}\right) \exp \left(-\frac{u_{n}^{2}(x)+u_{n}^{2}(y)}{4}\right) \\
< & 2 \sqrt{2} \mathbb{C}_{15} n\left(1-\rho_{n}\right)^{\frac{1}{2}} \exp \left(-\frac{b_{n}^{2}+x+y}{2}\right) \\
< & 8 \sqrt{\pi} \mathbb{C}_{15} b_{n}^{-2}\left(b_{n}^{3}\left(\log b_{n}\right)\left(1-\rho_{n}\right)^{\frac{1}{2}}\right) \\
< & b_{n}^{-2}
\end{aligned}
$$

due to $\lim _{\rho_{n} \rightarrow 1}\left(1-\rho_{n}\right)^{-1 / 2}\left(\pi / 2-\arcsin \left(\rho_{n}\right)\right)=\sqrt{2}$ and $\lim _{n \rightarrow \infty} b_{n}^{10}\left(1-\rho_{n}\right)=c$, where $\mathbb{C}_{15}$ is an absolute positive constant. Combining (i) with (A.21), we can obtain the upper bound in (2.1).

Finally, we consider the lower bound in (2.1). For fixed $x, y \in \mathbb{R}$, if $\max (x, y)<z<4 \log b_{n}$ we have

$$
\begin{aligned}
& \Phi\left(\frac{u_{n}(\min (x, y))-\rho_{n} u_{n}(z)}{\sqrt{1-\rho_{n}^{2}}}\right) \\
< & \frac{\exp \left(-\frac{b_{n}^{2}\left(1-\rho_{n}\right)}{4}-\frac{\min (x, y)}{1+\rho_{n}}+\frac{\rho_{n} z}{1+\rho_{n}}\right)}{\sqrt{2 \pi}\left(\frac{z-\min (x, y)}{2 \lambda_{n}}-\lambda_{n}-\frac{\lambda_{n} z}{b_{n}^{2}}\right)}
\end{aligned}
$$

for large $n$. So, for $\max (x, y)<z<4 \log b_{n}$,

$$
\begin{aligned}
& \int_{\max (x, y)}^{4 \log b_{n}} \Phi\left(\frac{u_{n}(\min (x, y))-\rho_{n} u_{n}(z)}{\sqrt{1-\rho_{n}^{2}}}\right) e^{-z} \exp \left(-\frac{z^{2}}{2 b_{n}^{2}}\right) d z \\
& <\frac{b_{n} \sqrt{1-\rho_{n}} \exp \left(-\frac{b_{n}^{2}\left(1-\rho_{n}\right)}{4}-\frac{\min (x, y)}{1+\rho_{n}}-\frac{\max (x, y)}{1+\rho_{n}}\right)}{\sqrt{\pi}\left(\max (x, y)-\min (x, y)-2 \lambda_{n}^{2}-\frac{8 \lambda_{n}^{2} \log b_{n}}{b_{n}^{2}}\right)} \\
& =O\left(b_{n}^{-4}\right)
\end{aligned}
$$

for large $n$ due to $\lim _{n \rightarrow \infty} b_{n}^{10}\left(1-\rho_{n}\right)=c$.

Note that

$$
\begin{aligned}
& \int_{4 \log b_{n}}^{\infty} \Phi\left(\frac{u_{n}(\min (x, y))-\rho_{n} u_{n}(z)}{\sqrt{1-\rho_{n}^{2}}}\right) e^{-z} \exp \left(-\frac{z^{2}}{2 b_{n}^{2}}\right) d z \\
= & O\left(b_{n}^{-4}\right)
\end{aligned}
$$

for large $n$. By (A.16), (3.36) and (3.37), we have

$$
\begin{aligned}
& 1-F\left(u_{n}(x), u_{n}(y)\right) \\
= & n^{-1} \int_{\max (x, y)}^{\infty} \Phi\left(\frac{u_{n}(\min (x, y))-\rho_{n} u_{n}(z)}{\sqrt{1-\rho_{n}^{2}}}\right) e^{-z} \\
& \times \exp \left(-\frac{z^{2}}{2 b_{n}^{2}}\right) d z+1-\Phi\left(u_{n}(\min (x, y))\right) \\
= & n^{-1}\left(-b_{n}^{-2} e^{-\min (x, y)}\left((\min (x, y))^{2} / 2+\min (x, y)+1\right)\right. \\
& \left.+e^{-\min (x, y)}+O\left(b_{n}^{-4}\right)\right)
\end{aligned}
$$

which implies

$$
\begin{aligned}
& F^{n}\left(u_{n}(x), u_{n}(y)\right)-H_{0}(x, y) \\
= & b_{n}^{-2}\left(\frac{(\min (x, y))^{2}}{2}+\min (x, y)+1+O\left(b_{n}^{-2}\right)\right) \\
& \times H_{0}(x, y) e^{-\min (x, y)}
\end{aligned}
$$

for large $n$. Hence the lower bound in (2.1) is obtained if $\rho_{n} \in(0,1)$ such that $\lim _{n \rightarrow \infty} b_{n}^{10}\left(1-\rho_{n}\right)=c$.

The proof is complete.

\section{APPENDIX A}

Auxiliary lemmas used in the proofs of the main results are given in this appendix.

Lemma 1. Let norming constant $b_{n}$ be given by (1.3). Under the second-order Hüsler-Reiss condition (1.5), for fixed $x, y \in \mathbb{R}$ we have

$$
b_{n}^{2} \int_{y}^{\infty}\left(\Phi\left(\frac{u_{n}(x)-\rho_{n} u_{n}(z)}{\sqrt{1-\rho_{n}^{2}}}\right)-\Phi\left(\lambda+\frac{x-z}{2 \lambda}\right)\right) e^{-z} d z
$$


(A.1)

$$
\begin{aligned}
= & \left(A_{n}+B_{n} x\right) \int_{y}^{\infty} \varphi\left(\lambda+\frac{x-z}{2 \lambda}\right) e^{-z} d z \\
& +\left(C_{n}-B_{n}\right) \int_{y}^{\infty} \varphi\left(\lambda+\frac{x-z}{2 \lambda}\right) z e^{-z} d z+O\left(b_{n}^{-2}\right)
\end{aligned}
$$

$$
\rightarrow-\kappa_{1}(x, y)
$$

as $n \rightarrow \infty$, and

$$
\begin{aligned}
& b_{n}^{2} \int_{y}^{\infty}\left(\Phi\left(\frac{u_{n}(x)-\rho_{n} u_{n}(z)}{\sqrt{1-\rho_{n}^{2}}}\right)-\Phi\left(\lambda+\frac{x-z}{2 \lambda}\right)\right) z^{2} e^{-z} d z \\
& =\left(A_{n}+B_{n} x\right) \int_{y}^{\infty} \varphi\left(\lambda+\frac{x-z}{2 \lambda}\right) z^{2} e^{-z} d z \\
& \quad+\left(C_{n}-B_{n}\right) \int_{y}^{\infty} \varphi\left(\lambda+\frac{x-z}{2 \lambda}\right) z^{3} e^{-z} d z+O\left(b_{n}^{-2}\right)
\end{aligned}
$$

$$
\rightarrow-\kappa_{2}(x, y)
$$

as $n \rightarrow \infty$, where $\varphi(x)$ denotes the standard Gaussian density function, and $\kappa_{1}(x, y), \kappa_{2}(x, y)$ are respectively given by

$$
\begin{aligned}
\kappa_{1}(x, y)= & \left(2 \lambda^{4}-2 \lambda^{2} x\right) e^{-x}\left(1-\Phi\left(\lambda+\frac{y-x}{2 \lambda}\right)\right) \\
& -\left(2 \alpha+3 \lambda^{3}\right) e^{-x} \varphi\left(\lambda+\frac{y-x}{2 \lambda}\right)
\end{aligned}
$$

and

$$
\begin{aligned}
\kappa_{2}(x, y)=( & 8 \lambda^{8}+32 \lambda^{6}-16 \lambda^{6} x+10 \lambda^{4} x^{2}-20 \lambda^{4} x+16 \alpha \lambda^{3} \\
& \left.-2 \lambda^{2} x^{3}-8 \alpha \lambda x\right) e^{-x}\left(1-\Phi\left(\lambda+\frac{y-x}{2 \lambda}\right)\right) \\
- & \left(8 \lambda^{7}+24 \lambda^{5}-4 \lambda^{5} y-12 \lambda^{5} x+4 \lambda^{3} x^{2}+4 \lambda^{3} x y\right. \\
& \left.+3 \lambda^{3} y^{2}+16 \alpha \lambda^{2}+2 \alpha y^{2}\right) e^{-x} \varphi\left(\lambda+\frac{y-x}{2 \lambda}\right) .
\end{aligned}
$$

Proof: By Taylor expansion with the Lagrange remainder term, we have

$$
\text { (A.5) } \begin{aligned}
& \left(\frac{u_{n}(x)-\rho_{n} u_{n}(z)}{\sqrt{1-\rho_{n}^{2}}}\right) \\
= & \Phi\left(\lambda+\frac{x-z}{2 \lambda}\right) \\
& +\varphi\left(\lambda+\frac{x-z}{2 \lambda}\right)\left(\frac{u_{n}(x)-\rho_{n} u_{n}(z)}{\sqrt{1-\rho_{n}^{2}}}-\lambda-\frac{x-z}{2 \lambda}\right) \\
& +\frac{1}{2} \nu_{n} \varphi\left(\nu_{n}\right)\left(\frac{u_{n}(x)-\rho_{n} u_{n}(z)}{\sqrt{1-\rho_{n}^{2}}}-\lambda-\frac{x-z}{2 \lambda}\right)^{2},
\end{aligned}
$$

$$
\begin{aligned}
= & \left(A_{n}+B_{n} x\right) \int_{y}^{\infty} \varphi\left(\lambda+\frac{x-z}{2 \lambda}\right) e^{-z} d z \\
& +\left(C_{n}-B_{n}\right) \int_{y}^{\infty} \varphi\left(\lambda+\frac{x-z}{2 \lambda}\right) z e^{-z} d z \\
\rightarrow & \left(\alpha+\frac{1}{2} \lambda^{3}-\frac{1}{2} \alpha \lambda^{-2} x+\frac{1}{4} \lambda x\right) \int_{y}^{\infty} \varphi\left(\lambda+\frac{x-z}{2 \lambda}\right) e^{-z} d z \\
& +\left(\frac{3}{4} \lambda+\frac{1}{2} \alpha \lambda^{-2}\right) \int_{y}^{\infty} \varphi\left(\lambda+\frac{x-z}{2 \lambda}\right) z e^{-z} d z \\
\text { A. } & 7) \\
= & \left(2 \lambda^{2} x-2 \lambda^{4}\right) e^{-x}\left(1-\Phi\left(\lambda+\frac{y-x}{2 \lambda}\right)\right) \\
& +\left(2 \alpha+3 \lambda^{3}\right) e^{-x} \varphi\left(\lambda+\frac{y-x}{2 \lambda}\right)
\end{aligned}
$$

and

$$
\begin{array}{r}
\int_{y}^{\infty} \varphi\left(\lambda+\frac{x-z}{2 \lambda}\right) e^{-z} d z=2 \lambda e^{-x}\left(1-\Phi\left(\lambda+\frac{y-x}{2 \lambda}\right)\right) \\
\int_{y}^{\infty} \varphi\left(\lambda+\frac{x-z}{2 \lambda}\right) z e^{-z} d z=4 \lambda^{2} e^{-x} \varphi\left(\lambda+\frac{y-x}{2 \lambda}\right) \\
+\left(2 \lambda x-4 \lambda^{3}\right) e^{-x}\left(1-\Phi\left(\lambda+\frac{y-x}{2 \lambda}\right)\right) .
\end{array}
$$

Combining with (3.2), we have

$$
\begin{aligned}
& b_{n}^{2} \int_{y}^{\infty}\left(\frac{u_{n}(x)-\rho_{n} u_{n}(z)}{\sqrt{1-\rho_{n}^{2}}}-\lambda-\frac{x-z}{2 \lambda}\right) \\
& \times \varphi\left(\lambda+\frac{x-z}{2 \lambda}\right) e^{-z} d z
\end{aligned}
$$

as $n \rightarrow \infty$. Similarly,

$$
\begin{aligned}
& b_{n}^{2} \int_{y}^{\infty}\left(\frac{u_{n}(x)-\rho_{n} u_{n}(z)}{\sqrt{1-\rho_{n}^{2}}}-\lambda-\frac{x-z}{2 \lambda}\right)^{2} \nu_{n} \varphi\left(\nu_{n}\right) e^{-z} d z \\
= & O\left(b_{n}^{-2}\right) .
\end{aligned}
$$

Combining with (A.6)-(A.8), we can derive (A.1) and (A.2).

By arguments similar to that of the first assertion, we can derive (A.3). The proof is complete.

Lemma 2. For large n, let

where $\min \left\{\frac{u_{n}(x)-\rho_{n} u_{n}(z)}{\sqrt{1-\rho_{n}^{2}}}, \lambda+\frac{x-z}{2 \lambda}\right\} \quad<\quad \nu_{n} \quad<\quad c_{n}:=\log \log b_{n}^{2}>0, \quad d_{n}:=-\log \log \frac{b_{n}^{2}}{b_{n}^{2}-1}>0$ 
with $b_{n}$ given by (1.3). Then under the second-order Hüsler-Reiss condition (1.5), with absolute positive constants $\mathbb{C}_{i}, 1 \leq i \leq 5$, we have

(1) for large $n$, the following inequality

$$
\begin{aligned}
& \int_{y}^{\infty}\left|\Phi\left(\frac{u_{n}(x)-\rho_{n} u_{n}(z)}{\sqrt{1-\rho_{n}^{2}}}\right)-\Phi\left(\lambda+\frac{x-z}{2 \lambda}\right)\right| e^{-z} d z \\
\leq & b_{n}^{-2}\left(e^{-y}\left(\mathbb{C}_{1}|y|+\mathbb{C}_{2}\right)+\mathbb{C}_{3} e^{-x}|x|+\mathbb{C}_{4}\right)
\end{aligned}
$$

holds uniformly for all $(x, y) \in\left[-c_{n}, d_{n}\right] \times\left[-c_{n}, d_{n}\right]$.

(2) for large $n$, the following inequality

(A.10)

$$
\begin{aligned}
& \int_{y}^{\infty}\left|\Phi\left(\frac{u_{n}(x)-\rho_{n} u_{n}(z)}{\sqrt{1-\rho_{n}^{2}}}\right)-\Phi\left(\lambda+\frac{x-z}{2 \lambda}\right)\right| e^{-z} d z \\
& \leq \mathbb{C}_{5} b_{n}^{-2}
\end{aligned}
$$

holds uniformly for all $(x, y) \in\left[-c_{n}, d_{n}\right] \times\left[d_{n}, \infty\right)$.

Proof: By the Taylor expansion with the Lagrange remainder, we have

(A.11)

$$
\begin{aligned}
& \int_{y}^{\infty}\left|\Phi\left(\frac{u_{n}(x)-\rho_{n} u_{n}(z)}{\sqrt{1-\rho_{n}^{2}}}\right)-\Phi\left(\lambda+\frac{x-z}{2 \lambda}\right)\right| e^{-z} d z \\
= & \int_{y}^{\infty}\left|\frac{u_{n}(x)-\rho_{n} u_{n}(z)}{\sqrt{1-\rho_{n}^{2}}}-\lambda-\frac{x-z}{2 \lambda}\right| \varphi\left(\nu_{n}\right) e^{-z} d z \\
\leq & b_{n}^{-2}\left(\left|A_{n}\right| \int_{y}^{\infty} \varphi\left(\nu_{n}\right) e^{-z} d z+\left|B_{n}\right||x| \int_{y}^{\infty} \varphi\left(\nu_{n}\right) e^{-z} d z\right. \\
& \left.+\left(\left|B_{n}\right|+\left|C_{n}\right|\right) \int_{y}^{\infty} \varphi\left(\nu_{n}\right)|z| e^{-z} d z\right) \\
\leq & b_{n}^{-2}\left(\left|A_{n}\right| \int_{y}^{\infty} e^{-z} d z+\left|B_{n}\right||x| \int_{y}^{d_{n}} \varphi\left(\nu_{n}\right) e^{-z} d z\right. \\
& \left.+\left|B_{n}\right||x| \int_{d_{n}}^{\infty} e^{-z} d z+\left(\left|B_{n}\right|+\left|C_{n}\right|\right) \int_{y}^{\infty}|z| e^{-z} d z\right) \\
\leq & 2 b_{n}^{-2}\left(\left|\frac{1}{2} \alpha \lambda^{-2}-\frac{1}{4} \lambda\right||x| \int_{y}^{d_{n}} \varphi\left(\nu_{n}\right) e^{-z} d z\right. \\
& +\left(\left|\frac{1}{2} \alpha \lambda^{-2}-\frac{1}{4} \lambda\right|+\lambda\right)\left(2-(y+1) e^{-y}\right) \\
& \left.+\left|\frac{1}{2} \lambda^{3}+\alpha\right| e^{-y}+\left|\frac{1}{2} \alpha \lambda^{-2}-\frac{1}{4} \lambda\right||x| e^{-d_{n}}\right)
\end{aligned}
$$

for large $n$, where $\min \left(\frac{u_{n}(x)-\rho_{n} u_{n}(z)}{\sqrt{1-\rho_{n}^{2}}}, \lambda+\frac{x-z}{2 \lambda}\right)<\nu_{n}<$ $\max \left(\frac{u_{n}(x)-\rho_{n} u_{n}(z)}{\sqrt{1-\rho_{n}^{2}}}, \lambda+\frac{x-z}{2 \lambda}\right)$. Note that, with the secondorder Hüsler-Reiss condition (1.5), we have

$$
\left|\frac{u_{n}(x)-\rho_{n} u_{n}(z)}{\sqrt{1-\rho_{n}^{2}}}-\lambda-\frac{x-z}{2 \lambda}\right|
$$

$$
\begin{aligned}
\leq & \left(c_{n}+d_{n}\right)\left|\frac{1}{\lambda_{n}}\left(1+\frac{\lambda_{n}^{2}}{2 b_{n}^{2}}+O\left(b_{n}^{-4}\right)\right)-\frac{1}{\lambda}\right| \\
& +\left|\lambda_{n}\left(1-\frac{\lambda_{n}^{2}}{b_{n}^{2}}\right)^{-\frac{1}{2}}-\lambda\right|+\frac{\lambda_{n}\left(c_{n}+d_{n}\right)}{b_{n}^{2}}\left(1-\frac{\lambda_{n}^{2}}{b_{n}^{2}}\right)^{-\frac{1}{2}} \\
\leq & \left|\lambda_{n}\left(1-\frac{\lambda_{n}^{2}}{b_{n}^{2}}\right)^{-\frac{1}{2}}-\lambda\right|+\frac{\lambda_{n}\left(c_{n}+d_{n}\right)}{b_{n}^{2}}\left(1-\frac{\lambda_{n}^{2}}{b_{n}^{2}}\right)^{-\frac{1}{2}} \\
& +\left(c_{n}+d_{n}\right) \frac{\left|\lambda-\lambda_{n}\right|}{\lambda \lambda_{n}}+\frac{\left(c_{n}+d_{n}\right) \lambda_{n}}{2 b_{n}^{2}}+O\left(\frac{c_{n}+d_{n}}{b_{n}^{4}}\right) \\
\rightarrow & 0
\end{aligned}
$$

uniformly for all $(x, z) \in\left[-c_{n}, d_{n}\right] \times\left[-c_{n}, d_{n}\right]$ as $n \rightarrow \infty$, which implies that $\varphi\left(\nu_{n}\right)$ converges to $\varphi\left(\lambda+\frac{x-z}{2 \lambda}\right)$ uniformly for $(x, z) \in\left[-c_{n}, d_{n}\right] \times\left[-c_{n}, d_{n}\right]$ since $|\varphi(x)-\varphi(y)|<$ $|x-y|$ for all $x, y \in \mathbb{R}$. Hence,

$$
\begin{aligned}
\int_{y}^{d_{n}} \varphi\left(\nu_{n}\right) e^{-z} d z & <2 \int_{y}^{d_{n}} \varphi\left(\lambda+\frac{x-z}{2 \lambda}\right) e^{-z} d z \\
& =2 e^{-x} \int_{y}^{d_{n}} \varphi\left(\lambda+\frac{z-x}{2 \lambda}\right) d z \\
& <4 \lambda e^{-x}
\end{aligned}
$$

for $(x, y) \in\left[-c_{n}, d_{n}\right] \times\left[-c_{n}, d_{n}\right]$. Combining with (A.11), we have

$$
\begin{aligned}
& \int_{y}^{\infty}\left|\Phi\left(\frac{u_{n}(x)-\rho_{n} u_{n}(z)}{\sqrt{1-\rho^{2}}}\right)-\Phi\left(\lambda+\frac{x-z}{2 \lambda}\right)\right| e^{-z} d z \\
\leq & b_{n}^{-2}\left(\left(\mathbb{C}_{1}+\mathbb{C}_{2}|y|\right) e^{-y}+\mathbb{C}_{3}|x| e^{-x}+\mathbb{C}_{4}\right)
\end{aligned}
$$

for large $n$, which completes the proof of (A.9).

For $(x, y) \in\left[-c_{n}, d_{n}\right] \times\left[d_{n}, \infty\right]$, we can derive the desired result immediately by (A.11) since

$$
\int_{y}^{\infty}\left|\Phi\left(\frac{u_{n}(x)-\rho_{n} u_{n}(z)}{\sqrt{1-\rho^{2}}}\right)-\Phi\left(\lambda+\frac{x-z}{2 \lambda}\right)\right| e^{-z} d z \leq \mathbb{C}_{5} b_{n}^{-2}
$$

for large $n$. The proof is complete.

Lemma 3. Let norming constant $b_{n}$ be defined by (1.3). Assume that the second-order Hüsler-Reiss condition (1.5) holds. Then for fixed $x, y \in \mathbb{R}$ and sufficiently large $n$, we have

$$
\begin{aligned}
& F^{n}\left(u_{n}(x), u_{n}(y)\right)-H_{\lambda}(x, y) \\
= & H_{\lambda}(x, y) b_{n}^{-2}\left(e^{-x}\left(1+x+\frac{1}{2} x^{2}\right)+\frac{1}{2} \kappa_{3}(x, y)\right. \\
& -\left(A_{n}+B_{n} x\right) \int_{y}^{\infty} \varphi\left(\lambda+\frac{x-z}{2 \lambda}\right) e^{-z} d z \\
& \left.+\left(B_{n}-C_{n}\right) \int_{y}^{\infty} \varphi\left(\lambda+\frac{x-z}{2 \lambda}\right) z e^{-z} d z+O\left(b_{n}^{-2}\right)\right),
\end{aligned}
$$


where

$$
\begin{aligned}
& \kappa_{3}(x, y)=\left(y^{2}+2 y+2\right) e^{-y} \Phi\left(\lambda+\frac{x-y}{2 \lambda}\right) \\
& -\left(4 \lambda^{4}-4 \lambda^{2} x+x^{2}+2 x+2\right) e^{-x}\left(1-\Phi\left(\lambda+\frac{y-x}{2 \lambda}\right)\right) \\
& +\left(4 \lambda^{3}-2 \lambda x-2 \lambda y-4 \lambda\right) e^{-x} \varphi\left(\lambda+\frac{y-x}{2 \lambda}\right) .
\end{aligned}
$$

Proof: First note that

$$
\begin{aligned}
& \int_{y}^{\infty} \Phi\left(\frac{u_{n}(x)-\rho_{n} u_{n}(z)}{\sqrt{1-\rho_{n}^{2}}}\right) e^{-z} \exp \left(-\frac{z^{2}}{2 b_{n}^{2}}\right) d z \\
= & \int_{y}^{\infty} \Phi\left(\frac{u_{n}(x)-\rho_{n} u_{n}(z)}{\sqrt{1-\rho_{n}^{2}}}\right) e^{-z}\left(1-\frac{z^{2}}{2 b_{n}^{2}}\right) d z+O\left(b_{n}^{-4}\right)
\end{aligned}
$$

holds for large $n$ due to $\left|e^{-x}-(1-x)\right|<x^{2} / 2$ for $x>0$.

Noting that

$$
\kappa_{3}(x, y)=\int_{y}^{\infty} \Phi\left(\lambda+\frac{x-z}{2 \lambda}\right) z^{2} e^{-z} d z
$$

and by (A.1) and (A.3), we have

$$
\begin{aligned}
& \int_{y}^{\infty} \Phi\left(\frac{u_{n}(x)-\rho_{n} u_{n}(z)}{\sqrt{1-\rho_{n}^{2}}}\right) e^{-z}\left(1-\frac{z^{2}}{2 b_{n}^{2}}\right) d z \\
= & \int_{y}^{\infty} \Phi\left(\lambda+\frac{x-z}{2 \lambda}\right) e^{-z} d z-2^{-1} b_{n}^{-2} \kappa_{3}(x, y) \\
& +\int_{y}^{\infty}\left(\Phi\left(\frac{u_{n}(x)-\rho_{n} u_{n}(z)}{\sqrt{1-\rho_{n}^{2}}}\right)\right. \\
& \left.-\Phi\left(\lambda+\frac{x-z}{2 \lambda}\right)\right) e^{-z} d z \\
- & \frac{b_{n}^{-2}}{2} \int_{y}^{\infty}\left(\Phi\left(\frac{u_{n}(x)-\rho_{n} u_{n}(z)}{\sqrt{1-\rho_{n}^{2}}}\right)\right. \\
& \left.-\Phi\left(\lambda+\frac{x-z}{2 \lambda}\right)\right) z^{2} e^{-z} d z \\
= & \int_{y}^{\infty} \Phi\left(\lambda+\frac{x-z}{2 \lambda}\right) e^{-z} d z-2^{-1} b_{n}^{-2} \kappa_{3}(x, y) \\
& +b_{n}^{-2}\left(A_{n}+B_{n} x\right) \int_{y}^{\infty} \varphi\left(\lambda+\frac{x-z}{2 \lambda}\right) e^{-z} d z \\
& +b_{n}^{-2}\left(C_{n}-B_{n}\right) \int_{y}^{\infty} \varphi\left(\lambda+\frac{x-z}{2 \lambda}\right) z e^{-z} d z+O\left(b_{n}^{-4}\right)
\end{aligned}
$$

for large $n$.

It follows from [7] that

(A.16)

$$
1-\Phi\left(u_{n}(x)\right)=n^{-1} e^{-x}\left[1-b_{n}^{-2}\left(1+x+\frac{1}{2} x^{2}\right)+O\left(b_{n}^{-4}\right)\right] \quad \sup _{(x, y) \in\left(-\infty,-c_{n}\right] \times \mathbb{R}} F^{n}\left(u_{n}(x), u_{n}(y)\right)
$$

for large $n$. Combining (1.3) and (A.14)-(A.16), we have

$$
\begin{aligned}
& 1-F\left(u_{n}(x), u_{n}(y)\right) \\
= & n^{-1} \int_{y}^{\infty} \Phi\left(\frac{u_{n}(x)-\rho_{n} u_{n}(z)}{\sqrt{1-\rho_{n}^{2}}}\right) e^{-z} \exp \left(-\frac{z^{2}}{2 b_{n}^{2}}\right) d z \\
& +1-\Phi\left(u_{n}(x)\right) \\
= & n^{-1}\left(\int_{y}^{\infty} \Phi\left(\frac{u_{n}(x)-\rho_{n} u_{n}(z)}{\sqrt{1-\rho_{n}^{2}}}\right) e^{-z}\left(1-\frac{z^{2}}{2 b_{n}^{2}}\right) d z\right. \\
& \left.+O\left(b_{n}^{-4}\right)\right)+1-\Phi\left(u_{n}(x)\right) \\
= & n^{-1}\left[e^{-x}-b_{n}^{-2} e^{-x}\left(1+x+\frac{1}{2} x^{2}\right)\right. \\
& +\int_{y}^{\infty} \Phi\left(\lambda+\frac{x-z}{2 \lambda}\right) e^{-z} d z-2^{-1} b_{n}^{-2} \kappa_{3}(x, y) \\
& +b_{n}^{-2}\left(A_{n}+B_{n} x\right) \int_{y}^{\infty} \varphi\left(\lambda+\frac{x-z}{2 \lambda}\right) e^{-z} d z \\
& \left.+b_{n}^{-2}\left(C_{n}-B_{n}\right) \int_{y}^{\infty} \varphi\left(\lambda+\frac{x-z}{2 \lambda}\right) z e^{-z} d z+O\left(b_{n}^{-4}\right)\right]
\end{aligned}
$$

for large $n$, which implies the desired result (A.12). The proof is complete.

Before proving Lemma 4, we cite the following distributional tail decomposition of standard Gaussian distribution in [1], i.e., for $x>0$,

$$
\begin{aligned}
1-\Phi(x) & =x^{-1} \varphi(x)-r(x) \\
& =x^{-1}\left(1-x^{-2}\right) \varphi(x)+s(x),
\end{aligned}
$$

where

$$
0<r(x)<x^{-3} \varphi(x), \quad 0<s(x)<3 x^{-5} \varphi(x) .
$$

Lemma 4. Let norming constant $b_{n}$ be defined by (1.3). For sufficiently large n, we have

$$
\sup _{(x, y) \in\left(-\infty,-c_{n}\right] \times \mathbb{R}}\left|\Delta\left(F^{n}, H_{\lambda} ; x, y\right)\right|<\mathbb{D}_{1} b_{n}^{-2},
$$

where $c_{n}=\log \log b_{n}^{2}$ and $\mathbb{D}_{1}$ is an absolute positive constant.

Proof: By (1.3), (A.18), (A.19) and $e^{z}>1+z, z \in \mathbb{R}$, for all $y \in \mathbb{R}$ we have

$$
\begin{aligned}
& 1-F\left(u_{n}\left(-c_{n}\right), u_{n}(y)\right) \geq 1-\Phi\left(u_{n}\left(-c_{n}\right)\right) \\
> & n^{-1}\left(\log b_{n}^{2}\right)\left(1-\frac{\left(\log \log b_{n}^{2}\right)^{2}}{2 b_{n}^{2}}-b_{n}^{-2}\left(1-\frac{\log \log b_{n}^{2}}{b_{n}^{2}}\right)^{-2}\right)
\end{aligned}
$$

for large $n$, which implies that

Convergence rate of maxima of bivariate Gaussian arrays to the Hüsler-Reiss distribution 361 


$$
\begin{aligned}
& \leq \sup _{y \in \mathbb{R}} F^{n}\left(u_{n}\left(-c_{n}\right), u_{n}(y)\right) \\
& \leq\left[1-\frac{\log b_{n}^{2}}{n}\left(1-\frac{\left(\log \log b_{n}^{2}\right)^{2}}{2 b_{n}^{2}}-\frac{1}{b_{n}^{2}\left(1-\frac{\log \log b_{n}^{2}}{b_{n}^{2}}\right)^{2}}\right)\right]^{n} \\
& \leq b_{n}^{-2} \exp \left(\frac{\left(\log b_{n}^{2}\right)\left(\log \log b_{n}^{2}\right)^{2}}{2 b_{n}^{2}}+\frac{\log b_{n}^{2}}{b_{n}^{2}\left(1-\frac{\log \log b_{n}^{2}}{b_{n}^{2}}\right)^{2}}\right) \\
& <\mathbb{C}_{6} b_{n}^{-2}
\end{aligned}
$$

for large $n$, where $\mathbb{C}_{6}$ is an absolute positive constant. Hence,

$$
\left|F^{n}\left(u_{n}(x), u_{n}(y)\right)-H_{\lambda}(x, y)\right|<\left(\mathbb{C}_{6}+1\right) b_{n}^{-2}=\mathbb{D}_{1} b_{n}^{-2}
$$

uniformly for all $(x, y) \in\left(-\infty,-c_{n}\right] \times \mathbb{R}$ for large $n$, which is the desired result.

\section{ACKNOWLEDGEMENTS}

The authors would like to thank the Editor-in-Chief and the referees for careful reading and for their perceptive and useful comments which greatly improved the paper. The authors appreciate the Guest Editor Prof. Zhengjun Zhang from University of Wisconsin at Madison, Prof. Liang Peng from Georgia Institute of Technology and Prof. Enkelejd Hashorva from University of Lausanne for their fruitful discussions on this topic. This work was supported by the National Natural Science Foundation of China grant no. 11171275, the Natural Science Foundation Project of CQ no. cstc2012jjA00029, and the Fundamental Research Funds for the Central Universities (XDJK2012C045, XDJK2014D020).

\section{Received 20 September 2013}

\section{REFERENCES}

[1] Abramowtiz, M. and Stegun, I. A. (1972). Handbook of Mathematical Functions. National Bureau of Standards, Washington, D.C. MR0757537

[2] de HaAn, L. and Peng, L. (1997). Rates of convergence for bivariate extremes. J. Multivariate Anal. 61 195-230. MR1452273

[3] DE HAAn, L. and Resnick, S. (1996). Second-order regular variation and rates of convergence in extreme value theory. Ann. Prob. 24 97-124. MR1387628

[4] Engelke, S., Kabluchko, Z., and Schlather M. (2014a). Maxima of independent, non-identically distributed Gaussian vectors. Bernoulli in press.

[5] Engelke, S., Malinowski, A., KabluchKo, Z., and Schlather, M. (2014b). Estimation of Hüsler-Reiss distributions and Brown-Resnick processes. J. R. Stat. Soc. Series B in press.

[6] Frick, M. and Reiss, R.-D. (2013). Expansions and penultimate distributions of maxima of bivariate normal random vectors. Statist. Probab. Lett. 83 2563-2568. MR3144042

[7] Hall, P. (1979). On the rate of convergence of normal extremes. J. Appl. Probab. 16 433-439. MR0531778
[8] Hall, W. J. and Wellner, J. A. (1979). The rate of convergence in law of the maximum of an exponential sample. Statist. Neerlandica 33 151-154. MR0552259

[9] Hashorva, E. (2005). Elliptical triangular arrays in the maxdomain of attraction of Hüsler-Reiss distribution. Statist. Probab. Lett. 72 125-135. MR2137118

[10] Hashorva, E. (2006). On the multivariate Hüsler-Reiss distribution attracting the maxima of elliptical triangular arrays. Statist. Probab. Lett. 76 2027-2035. MR2329248

[11] Hashorva, E., Kabluchko, Z., and WüBker, A. (2012). Extremes of independent chi-square random vectors. Extremes $\mathbf{1 5}$ 35-42. MR2891308

[12] Hashorva, E. and Weng, Z. (2013). Limit laws for extremes of dependent stationary Gaussian arrays. Statist. Probab. Lett. 83 320-330. MR2998759

[13] Hashorva, E., Peng, Z., and Weng, Z. (2013). Higher-order expansions on distributions of bivariate extremes of Hüsler-Reiss model. Methodol. Comput. Appl. Probab. doi: 10.1007/s11009014-9407-6.

[14] Hooghiemstra, G. and Hüsler, J. (1996). A note on maxima of bivariate random vectors. Statist. Probab. Lett. 1 1-6. MR1421762

[15] HüsLER, J. (1994). Maxima of bivariate random vectors: Between independence and complete dependence. Statist. Probab. Lett. 21 385-394. MR1325215

[16] HüsleR, J. and Reiss, R.-D. (1989). Maxima of normal random vectors: Between independence and complete dependence. Statist. Probab. Lett. 7 283-286. MR0980699

[17] Kabluchko, Z., De HaAn, L., and Schlatter, M. (2009). Stationary max-stable fields associated to negative definite functions. Annals of Probability 37 2042-2065. MR2561440

[18] Leadbetter, M. R., Lindgren, G., and Rootzén, H. (1983). Extremes and Related Properties of Random Sequences and Processes. Springer Verlag, New York. MR0691492

[19] Liao, X. and Peng, Z. (2012). Convergence rates of limit distribution of maxima of lognormal samples. J. Math. Anal. Appl. 395 643-653. MR2948256

[20] Li, W. V. and Shao, Q. M. (2002). A normal comparison inequality and its applications. Probab. Theory Related Fields $\mathbf{1 2 2}$ 494-508. MR1902188

[21] Peng, Z., Nadarajah, S., and Lin, F. (2010). Convergence rate of extremes for the general error distribution. J. Appl. Probab. 47 668-679. MR2731341

[22] Resnick, S. I. (1987). Extreme Values, Regular Variation and Point Processes. Springer Verlag, New York. MR0900810

Xin Liao

Department of Statistics

School of Mathematics and Statistics

Southwest University

Chongqing, 400715

China

E-mail address: liaoxin2010@163.com

Zuoxiang Peng

Department of Statistics

School of Mathematics and Statistics

Southwest University

Chongqing, 400715

China

E-mail address: pzx@swu.edu.cn 